\title{
Iterative Power Allocation Algorithms for Amplify/Estimate/Compress-and-Forward Multi-Band Relay Channels
}

\author{
Kyounghwan Lee and Aylin Yener \\ Electrical Engineering Department \\ The Pennsylvania State University \\ University Park, PA 16802 \\ Telephone: (814) 865-4337 \\ Fax: (814) $863-5341$ \\ kxl251@psu.edu \\ yener@ee.psu.edu
}

\begin{abstract}
We investigate the optimum transmit power allocation problem for the multi-band relay (MBR) channel. MBR is defined by a source node, a relay node, and a destination node communicating over multiple orthogonal bands. We focus on three relay transmission schemes: amplify-and-forward (AF), estimate-and-forward (EF), and compress-and-forward (CF) and study the optimum transmit source and relay power allocations to maximize the instantaneous achievable rate. We formulate iterative algorithms to find the power allocation for each scheme. It is shown that $\mathrm{CF}$ is always better than $\mathrm{EF}$ and $\mathrm{AF}$. We observe that when the source-to-relay links are better than the sourceto-destination links, EF and AF perform equally well. When the source-to-relay links are worse than source-to-destination links, AF performs better than EF.
\end{abstract}

\section{INTRODUCTION}

Recently, relay networks have been attracting considerable attention for their potential of improving the performance of wireless systems. This performance gain is offered by exploiting the inherent broadcasting nature of the wireless channel and the spatial diversity in a distributed fashion. The earlier work on full-duplex relay channel by [1], [2] provides the theoretical background and hints the performance improvement over point-to-point communication. Since then, a significant research effort has been directed towards capacity and performance of relay networks in various set-ups [3]-[7].

Hindered by the difficulty of employing full-duplex at the relay node, most of the recent research efforts focus on orthogonal relay transmission schemes where the source and the relay node employ time-division [4], [6], [8]-[11], frequencydivision [12]-[15], or code-division [3]. It has been shown that a significant performance improvement can be achieved by optimum resource allocation, i.e., transmit power, bandwidth, or transmission time slot duration in these networks. [4], [9], [11], [12], [15]-[18].

The model considered in reference [12] employs two orthogonal frequency bands, one at the source and one at the relay. By employing the optimum bandwidth allocation, the reference shows that achievable rates employing decode-andforward can be improved over equal allocation. The optimum source and relay transmit power and time-slot allocation has been investigated for the three node half-duplex relay channel in reference [4]. The optimum relay transmit power allocation was investigated in [17] for various relaying transmission schemes for a relay assisted F/TDMA network with multiple source-destination pairs where all channels of all sources nodes and relay nodes are orthogonal. The bandwidth and power allocation for the relay networks where a single source and destination pair with multiple relay nodes employing amplify-and-forward and decode-and-forward schemes was investigated in [16]. In a similar setting, power allocation with partial channel side information was considered in [19], [20].

The so called multi-band relay channel (MBR) where the source and the relay nodes employ multiple orthogonal channels has been considered in [15]. The optimum source transmit power and bandwidth allocation improves the achievable rates of such networks employing decode-and-forward (DF) over power allocation only.

In this work, we investigate the jointly optimum transmit power allocation of the source and the relay node for MBR channel. We focus on three relay transmission schemes that do not decode the information at the relay: amplify-and-forward (AF), estimate-and-forward (EF), and compress-and-forward (CF). Our motivation for focusing on these three transmission schemes is their advantage over DF. AF is known to provide the same multiplexing gain and better diversity order than DF [6]. EF and CF transmission schemes outperform DF, especially when the source to the relay channel condition is worse than the source to the destination channel [4], [8].

We investigate the transmit source and relay power allocation problems that maximize the instantaneous achievable rate under individual power constraints. The solution for each scheme is reached by essentially the same iterative algorithm with the associated variables varying depending on the scheme. The proposed algorithms are convergent, and we observe numerically, the convergence to the optimum point.

We observe that when the source-to-relay links are better than the source-to-destination links, $\mathrm{EF}$ and $\mathrm{AF}$ perform equally well. When the source-to-relay links are worse than source-to-destination links, AF performs better than EF. CF always performs better than EF and AF. We also note the difference in the power allocation strategy between $\mathrm{CF}$ and $\mathrm{EF} / \mathrm{AF}$ : in $\mathrm{CF}$, the relay and source power allocation are 
independent, while in EF/AF, they affect and compensate each other to yield the maximum rate.

The rest of the paper is organized as follows. Section II presents system model. Section III derives the instantaneous mutual information for MBR of each scheme and investigates the corresponding iterative power allocation scheme. In Section IV, we present the numerical results. We conclude the paper in Section V.

\section{System ModeL}

We consider a three node relay network where multiple frequency bands available from the source and the relay are mutually orthogonal. We term this the multi-band relay (MBR) channel, which is given by Figure 1 . We assume that the source transmits information over several frequency channels, which is received by the destination and the relay. The relay forwards the received signal from the source after appropriate processing to the destination over the orthogonal frequency channels. The signal received by the relay and destination in the $i$ th orthogonal channel of the source node is given by

$$
\begin{aligned}
& y_{s d}^{i}=h_{s d}^{i} x_{s}^{i}+n_{s d}^{i} \\
& y_{s r}^{i}=h_{s r}^{i} x_{s}^{i}+n_{s r}^{i}
\end{aligned}
$$

where $x_{s}^{i}$ is the symbol transmitted by the source node in channel $i$ with transmit power, $p_{s}^{i}$. $n_{s d}^{i}$ and $n_{s r}^{i}$ are the independent additive white Gaussian (AWGN) noise with unit variance at the destination and the relay, respectively. We let $h_{s d}^{i}$ and $h_{s r}^{i}$ denote the channel gains from the source node to the destination node and the relay node in channel $i$.

Similarly, the signal received by the destination node in the second phase from channel $i$ is given by

$$
y_{r d}^{i}=h_{r d}^{i} x_{r}^{i}+n_{r d}^{i}
$$

where $x_{r}^{i}$ is the symbol transmitted by the relay node in channel $i$ with transmit power, $p_{r}^{i}$. $n_{r d}^{i}$ is the independent additive white Gaussian (AWGN) noise with unit variance at the destination. We let $h_{r d}^{i}$ denote the channel gain from the relay node to the destination node in channel $i$.

We consider three relay transmission schemes that does not decode the information received at the relay. In particular, we consider:

- Amplify-and-Forward (AF): The relay node forwards the scaled version of the noisy copy of the source signal it received to the destination node [6].

- Estimate-and-Forward (EF): The relay node forwards an estimate of its received signal to the destination node [2], [21].

- Compress-and-Forward (CF): The relay node compresses the received signal using Wyner-Ziv lossy source coding [22] and forwards to the destination [2], [23].

\section{Iterative Power Allocation Algorithm}

In this paper, we investigate the optimum source and relay transmit power allocation that maximizes the instantaneous achievable rate for three different relay schemes under individual power constraints. The resulting optimization problems

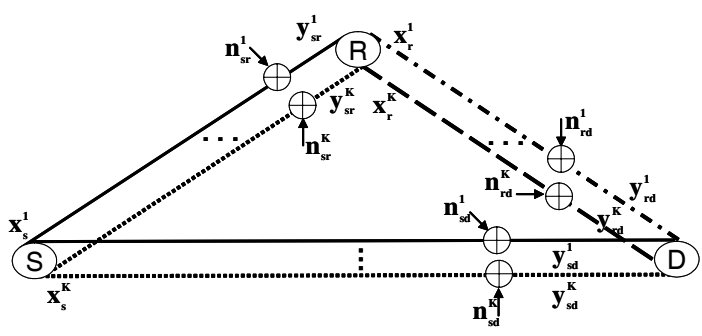

Fig. 1. Multi-Band Relay Channel

do not have closed form solutions. We thus construct iterative algorithms to converge to the optimum point of the corresponding problem.

The common theme is that for each of the three schemes we have an optimization problem that has identical characteristics, and we can come up with an alternating minimization based iterative algorithm to obtain the solution.

In particular, we find that, for $\mathrm{AF}, \mathrm{EF}$, and $\mathrm{CF}$, the updates that the iterative algorithm employs for each iteration are given by

$$
\begin{gathered}
\left(p_{r}^{i}\right)^{*}=\left(\frac{-r_{1}}{2 r_{2}}+\frac{1}{2} \sqrt{\left(\frac{r_{1}}{r_{2}}\right)^{2}-\frac{4 r_{0}}{r_{2}}}\right)^{+} \\
\left(p_{s}^{i}\right)^{*}=\left(-\frac{1}{s_{2}}+\left[R+\sqrt{Q^{3}+R^{2}}\right]^{1 / 3}+\left[R-\sqrt{Q^{3}+R^{2}}\right]^{1 / 3}\right)_{(5)}^{+}
\end{gathered}
$$

where $R$ and $Q$ are given by

$$
R=\frac{9 s_{1} s_{2}-27 s_{0}-2 s_{2}^{3}}{54}, \quad Q=\frac{3 s_{1}-s_{2}^{2}}{9}
$$

where $s_{2}, s_{1}, s_{0}, r_{2}, r_{1}$, and $r_{0}$ are given differently for each relay transmission scheme. The updates given by (4) and (5) are obtained simply from the first order optimality conditions for each channel. Thus, simply taking the derivative of the objective function for each schemes with respect to $p_{s}^{i}$ and $p_{r}^{i}$ and equating it to zero and after algebraic manipulation, we can obtain (4) and (5) that can be used for each of the three schemes. The parameters $s_{2}, s_{1}, s_{0}, r_{2}, r_{1}$, and $r_{0}$, need to be determined for each relay scheme.

The proposed iterative algorithm calls for optimizing one variable at a time while fixing the values of the rest. We start with random set of $p_{s}^{i}$ and $p_{r}^{i}$ and update each one of them by using (4) and (5) while keeping the transmit power constraints satisfied.

\section{A. Iterative Power Allocation for Amplify-and-Forward}

In amplify-and-forward (AF) scheme, the received signal at the relay over channel $i$ is amplified by the gain, $g_{i}$. Thus, the transmitted signal at the relay over channel $i$ is given by

$$
x_{r}^{i}=g_{i} y_{s r}^{i}
$$

where $g_{i}=\sqrt{\frac{P_{r}^{i}}{\left|h_{s r}^{i}\right|^{2} P_{s}^{i}+n_{s r}^{i}}}$. The amplifying gain, $g_{i}$, is used to maintain power constraints at the relay [6]. The maximization problem of MBR channel for AF can be stated as follows 


$$
\begin{array}{lc}
\max _{p_{s}^{i}, p_{r}^{i}} & \sum_{i=1}^{K} \frac{1}{2 K} \log \left(1+p_{s}^{i} a^{i}+\frac{p_{s}^{i} p_{r}^{i} b^{i} c^{i}}{1+b^{i} p_{s}^{i}+c^{i} p_{r}^{i}}\right) \\
\text { s. t. } & \sum_{i=1}^{K} p_{s}^{i}=P_{S}, \quad \sum_{i=1}^{K} p_{r}^{i}=P_{R}
\end{array}
$$

where we denote $a^{i}, b^{i}$, and $c^{i}$ as $\left|h_{s d}^{i}\right|^{2},\left|h_{s r}^{i}\right|^{2}$, and $\left|h_{r d}^{i}\right|^{2}$, respectively. $P_{S}$ and $P_{R}$ are the total transmit power constraints for the source and the relay node, respectively. $K$ is the total number of frequency channels for each communication phase. The factor $1 / 2 K$ accounts for the fact that information is conveyed over $2 K$ frequencies. The logarithm is base 2 . The index $i$ for $a^{i}, b^{i}$ and $c^{i}$ is omitted for conciseness. In this case, the parameters yielding iterations (4) and (5) are given by

$$
\begin{aligned}
& s_{2}=\frac{1+c p_{r}^{i}}{a}+\frac{2+2 c p_{r}^{i}}{b}-\frac{1}{2 K \lambda_{1}} \\
& s_{1}=\frac{2+3 c p_{r}^{i}+c^{2}\left(p_{r}^{i}\right)^{2}}{a b}+\frac{\left(c p_{r}^{i}+1\right)^{2}}{b^{2}}-\frac{2+2 c p_{r}^{i}}{2 K \lambda_{1} b} \\
& s_{0}=\frac{\left(c p_{r}^{i}+1\right)^{2}}{a b^{2}}-\frac{\left(c p_{r}^{i}+1\right)^{2}}{2 K \lambda_{1} b^{2}}-\frac{c p_{r}^{i}\left(1+c p_{r}^{i}\right)}{2 K \lambda_{1} a b} \\
& r_{2}=2 K \lambda_{2}\left(c^{2}+(a+b) c^{2} p_{s}^{i}\right) \\
& r_{1}=2 K \lambda_{2}\left(2 c+2 a b c\left(p_{s}^{i}\right)^{2}+(2 a c+3 b c) p_{s}^{i}+b^{2} c\left(p_{s}^{i}\right)^{2}\right) \\
& r_{0}=2 K \lambda_{2}\left(1+(a+2 b) p_{s}^{i}+\left(2 a b+b^{2}\right)\left(p_{s}^{i}\right)^{2}+a b^{2}\left(p_{s}^{i}\right)^{3}\right)-t_{i}
\end{aligned}
$$

where $t_{i}=b c p_{s}^{i}\left(1+b p_{s}^{i}\right) \cdot \lambda_{1}$ and $\lambda_{2}$ are the Lagrangian multipliers associated with the transmit power constraints of the source and the relay node, respectively.

\section{B. Iterative Power Allocation for Estimate-and-Forward}

In estimate-and-forward (EF), the relay node uses the estimate of the received signal at the relay. The achievable rate of EF is always greater than that of direct transmission [11]. On the other hand, the achievable rate of DF is less than that of direct transmission when the source to the destination channel condition is better than the source to the relay channel condition [11], making EF more attractive in this setting. For $\mathrm{EF}$, the maximization problem of MBR can be stated as

$$
\begin{array}{lc}
\max _{p_{s}^{i}, p_{r}^{i}} & \sum_{i=1}^{K} \frac{1}{2 K} \log \left(1+p_{s}^{i} a^{i}+\frac{p_{s}^{i} p_{r}^{i} b^{i} c^{i}}{1+\left(a^{i}+b^{i}\right) p_{s}^{i}+c^{i} p_{r}^{i}}\right) \\
\text { s. t. } & \sum_{i=1}^{K} p_{s}^{i}=P_{S}, \quad \sum_{i=1}^{K} p_{r}^{i}=P_{R}
\end{array}
$$

and the corresponding variables in (4) and (5) are given by

$$
\begin{aligned}
& s_{2}=\frac{1}{a}+2 \frac{a^{2}+a b+\left(a^{2} c+2 a b c+b^{2} c\right) p_{r}^{i}}{a^{3}+2 a^{2} b+a b^{2}}-\frac{1}{2 K \lambda_{1} a} \\
& s_{1}=\frac{3 a+2 b+(4 a+3 b) c p_{r}^{i}+(a+b)\left(c p_{r}^{i}\right)^{2}}{a^{3}+2 a^{2} b+a b^{2}}-\mu_{i} \\
& s_{0}=\frac{\left(2 K \lambda_{1}-1\right)\left(1+2 c p_{r}^{i}+c^{2}\left(p_{r}^{i}\right)^{2}\right)+b c p_{r}^{i}+b c^{2}\left(p_{r}^{i}\right)^{2}}{2 K \lambda_{1}\left(a^{3}+2 a^{2} b+a b^{2}\right)} \\
& r_{2}=2 K \lambda_{2} c^{2} \\
& r_{1}=2 K \lambda_{2}\left(2 c+(2 a c+b c) p_{s}^{i}\right) \\
& r_{0}=2 K \lambda_{2}\left(1+(2 a+b) p_{s}^{i}+\left(a^{2}+b^{2}\right)\left(p_{s}^{i}\right)^{2}\right)+b c p_{s}^{i}
\end{aligned}
$$

where $\mu_{i}=\frac{(a+b)\left(1+c p_{r}^{i}\right)}{K \lambda_{1}\left(a^{3}+2 a^{2} b+a b^{2}\right)}$.

\section{Iterative Power Allocation for Compress-and-Forward}

In compress-and-forward (CF), the relay node forwards a compressed version of its channel output to the destination node [2], [23]. This information is used as side information at the destination. This scheme achieves gains comparable to MIMO transmission [23]. The maximization problem of MBR for $\mathrm{CF}$ can be stated as follows

$$
\begin{array}{ll}
\max _{p_{s}^{i}, p_{r}^{i}} & \sum_{i=1}^{K} \frac{1}{2 K} \log \left(1+p_{s}^{i} a^{i}+\frac{b^{i} p_{s}^{i}}{1+\sigma_{W i}^{2}}\right) \\
\text { s. t. } & \sum_{i=1}^{K} p_{s}^{i}=P_{S}, \quad \sum_{i=1}^{K} p_{r}^{i}=P_{R}
\end{array}
$$

where $\sigma_{W i}^{2}$ is given by

$$
\sigma_{W i}^{2}=\frac{p_{s}^{i}\left(a^{i}+b^{i}\right)+1}{p_{r}^{i} c^{i}\left(p_{s}^{i} a^{i}+1\right)}
$$

Then, the corresponding variables are given by

$s_{2}=1+\frac{2(a+b)+c(2 a+b) p_{r}^{i}}{(a+b)^{2}+a c(a+b) p_{r}^{i}}+\frac{a^{2}(1+2 b)+a^{2} c(1+b) p_{r}^{i}}{2 K \lambda_{1}\left((a+b)^{2}+a c(a+b) p_{r}^{i}\right)}$

$s_{1}=\frac{2(a+b)+c(2 a+b+1) p_{r}^{i}}{(a+b)^{2}+a c(a+b) p_{r}^{i}}+\frac{2 a(a+b)\left(1+c p_{r}^{i}\right)}{2 K \lambda_{1}\left((a+b)^{2}+a c(a+b) p_{r}^{i}\right)}$

$s_{0}=\frac{2 K \lambda_{1}+a+c(a+b) p_{r}^{i}}{2 K \lambda_{1}\left((a+b)^{2}+a c(a+b) p_{r}^{i}\right)}$
$r_{2}=2 K \lambda_{2}\left(c^{2}+a c^{2} p_{s}^{i}\right)$
$r_{1}=2 K \lambda_{2}\left(2 c+(2 a+b) c p_{s}^{i}\right)$
$r_{0}=2 K \lambda_{2}\left(1+(a+b) p_{s}^{i}\right)+b c p_{s}^{i}$

\section{Convergence of Iterative Algorithm}

The objective function of the each relaying schemes are concave in each variable, but not jointly concave. First we note that each iteration of the algorithms discussed in this section increases the objective function as the function is concave in each variable. Also, the maximum achievable rate as a result of each optimization problem is bounded from above. Thus the proposed iterative algorithms are convergent. In addition, they are guaranteed to converge to the optimum point if it is unique [24].

In general, the nonconvexity of the problem prevents us from making a claim of convergence of the algorithm to the global optimum. As is common with nonconvex problems, we run multiple iteration by choosing multiple initial random transmit power and find the best of the points. Note that we are guaranteed global optimality if the channel gains satisfy the following condition:

$$
\mathbf{h}=\left[h_{s r}^{i}, h_{s d}^{i}, h_{r d}^{i}: \frac{d^{2} J}{d\left(p_{s}^{i}\right)^{2}} \frac{d^{2} J}{d\left(p_{r}^{i}\right)^{2}}-\left(\frac{d^{2} J}{d p_{s}^{i} d p_{r}^{i}}\right)^{2} \geq 0\right]
$$

where $i \in(1, K)$ and $J$ is the objective function of each scheme. This is because, for $\mathbf{h}$ satisfying (33), the objective 


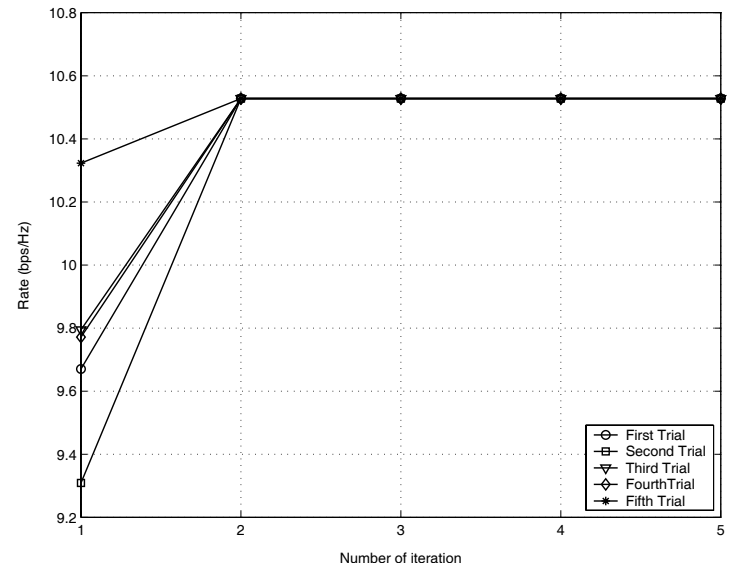

Fig. 2. Convergence of the iterative algorithm for AF scheme with five different random starting points

TABLE I

Four CASES DEPENDING ON CHANNEL CONDITIONS

\begin{tabular}{|l|c|c|c|c|}
\hline Case & I & II & III & IV \\
\hline \hline SNR of $\mathrm{SR}_{1}$ & $30 \mathrm{~dB}$ & $20 \mathrm{~dB}$ & $30 \mathrm{~dB}$ & $20 \mathrm{~dB}$ \\
\hline SNR of $\mathrm{SD}_{1}$ & $15 \mathrm{~dB}$ & $10 \mathrm{~dB}$ & $10 \mathrm{~dB}$ & $15 \mathrm{~dB}$ \\
\hline SNR of $\mathrm{SR}_{2}$ & $20 \mathrm{~dB}$ & $30 \mathrm{~dB}$ & $20 \mathrm{~dB}$ & $30 \mathrm{~dB}$ \\
\hline SNR of $\mathrm{SD}_{2}$ & $10 \mathrm{~dB}$ & $15 \mathrm{~dB}$ & $15 \mathrm{~dB}$ & $10 \mathrm{~dB}$ \\
\hline
\end{tabular}

functions become jointly concave and we can guarantee the convergence to the global optimum with over the convex constraint set, e.g, (25).

\section{Numerical Results}

In this section, we present numerical results to support our analysis. Specifically, we plot the maximum achievable rate for each of the three schemes and the corresponding transmit source and relay power allocation. For numerical results, the number of orthogonal channels from the source and the relay is each assumed to be two. We fix the source to the destination (SD) received SNRs and the source to the relay (SR) received SNRs while changing the relay to the destination (RD) received SNRs in each channel by the same amount.

Table I shows the four cases considered in numerical results. In case I, SR and SD links of channel 1 is better than that of channel 2. In case II, SR and SD links of channel 1 is worse than that of channel 2. In case III, SR link of channel 1 is better than that of channel 2 and SD link of channel 1 is worse than that of channel 2. In case IV, SR link of channel 1 is worse than that of channel 2 and SD link of channel 1 is better than that of channel 2. We note that SR links are better than SD links for all four cases.

Figure 2 shows the convergence of the proposed iterative algorithm to the optimum resource allocation for AF. Starting with random initial power allocation for each trial, we observe that the each time converges to the same value. Same observation is valid for $\mathrm{CF}$ and $\mathrm{EF}$ also. With random initial power allocation and channel gains, the iterative algorithm always converges to the optimum value.

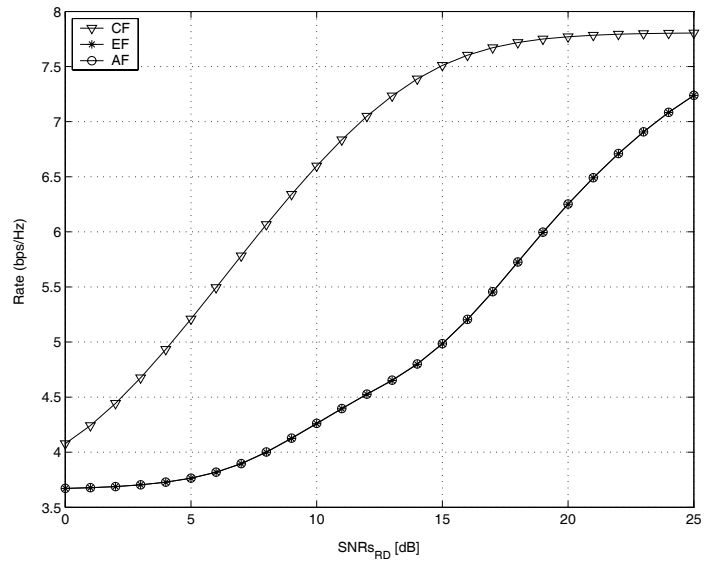

Fig. 3. Comparison of maximum achievable rates

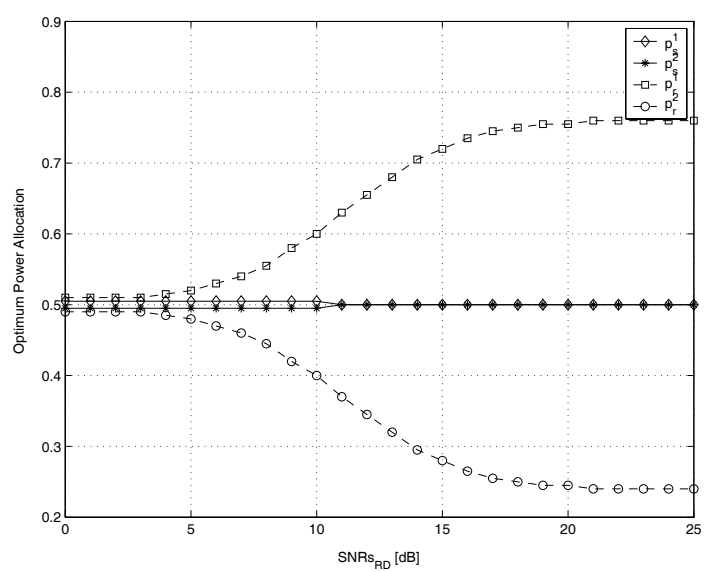

Fig. 4. Power Allocation for CF of case I

Figure 3 shows the maximized achievable rates of three relay transmission schemes with optimum power allocation for case I. Since the received SNRs of overall system are fixed as given in Table I, the maximized achievable rates for all four cases are identical. We can observe that $\mathrm{CF}$ is always better than EF and AF. Also, when SR links are better than SD links, $\mathrm{EF}$ and AF perform equally well.

Figure 4 shows the transmit source and relay power allocation of $\mathrm{CF}$ scheme for case I. We observe that almost equal source power is allocated to the SR/SD links. A slightly more source power is allocated to the channel with higher SD received SNR. For relay power allocation, as RD received SNRs become better, more relay power is allocated to the channel with higher SR received SNR. For better SR link, the relay node can provide the more useful side information to the destination. Thus, allocating more relay power to the channel with higher SR received SNR can help the reliable decodability at the destination node in CF scheme.

Figure 5 shows the transmit source and relay power allocation of EF/AF scheme for case I. In this case, the power allocation strategies between two schemes are same. At the low RD received SNRs, more source power is allocated to 


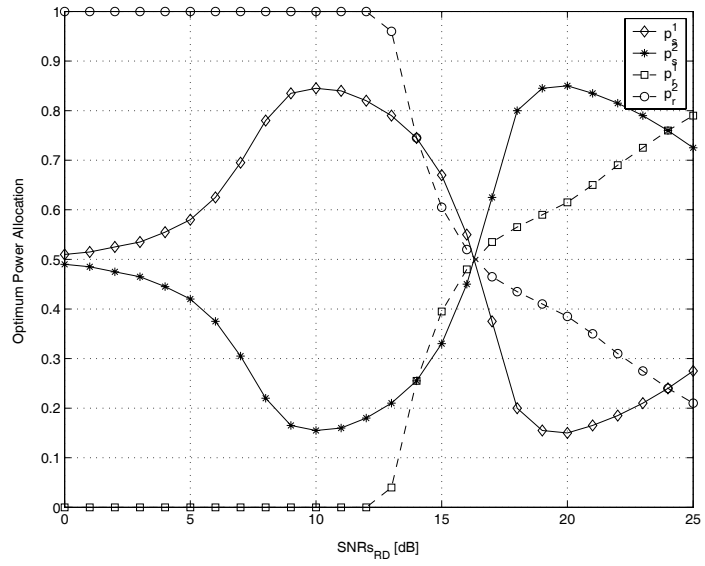

Fig. 5. Power Allocation for EF/AF of case I

the channel with larger SD received SNR because in this case, relay is not going to be helpful and allocating more source power to the channel with better SD received SNR yields higher rate. As the RD received SNRs become better, more source power is allocated to the channel with smaller $\mathrm{SR}$ received SNR. This is to ensure that relay node can be helpful for that link. That is, allocating more source power to the channel with smaller SR received SNR will help the relay receive the correct message from the source. With the relatively smaller power, the link with higher SNR can do the same. For relay power allocation, more relay power is allocated to the channel with smaller SR received SNR at low $\mathrm{RD}$ received SNRs. As the RD received SNRs become better, more relay power is allocated to the channel with larger SR received SNR.

For case II, since the channel conditions are opposite to the case I, the source and relay power allocation strategy is the same with case I with changing the role of channel 1 and channel 2.

Figure 6 shows the transmit source and relay power allocation of $\mathrm{CF}$ scheme for case III. We observe that almost equal source power is allocated to the SR/SD links. A slightly more source power is allocated to the channel with larger SD received SNR. For relay power allocation, as RD received SNRs become better, more relay power is allocated to the channel with larger SR received SNR. We note that the power allocation of CF for case III is almost identical to that of case I except for the role change between $P_{s}^{1}$ and $P_{s}^{2}$ because case III is identical to case I with switching the role of SR channel 1 and 2.

Figure 7 shows the transmit source and relay power allocation of EF/AF scheme for case III. In this case, the power allocation strategies between two schemes are same. At the low RD received SNR, more source power is allocated to the channel with worse SD link. As the RD received SNRs becomes better, more power is allocated to the channel with better SR received SNR. For relay power allocation, more relay power is allocated to the channel with larger SR received SNR at low RD received SNRs. As the RD received SNRs become better, relay power starts to be allocated to the channel

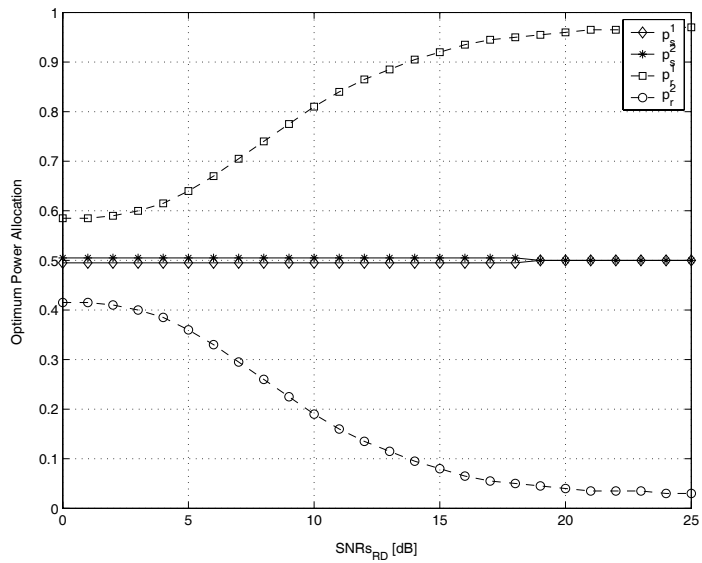

Fig. 6. Power Allocation for CF of case III

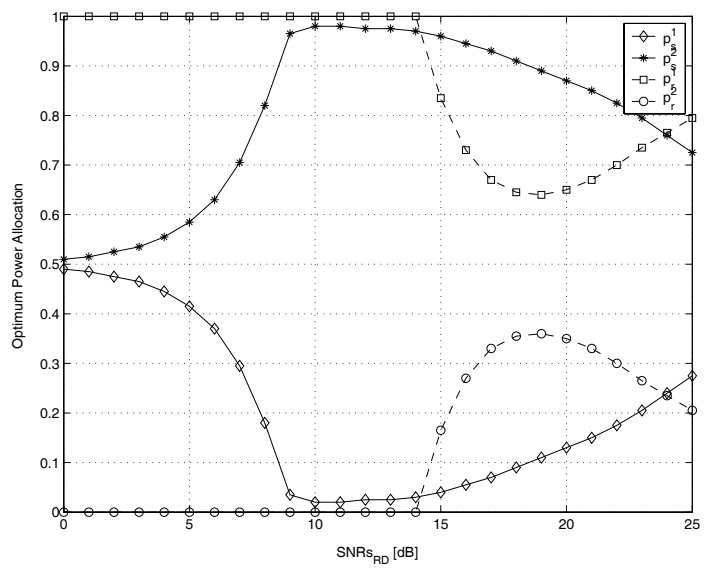

Fig. 7. Power Allocation for EF/AF of case III

with worse SR links. For case IV, since the channel conditions are opposite to the case III, the source and relay power allocation strategy is the same with case III with changing the role of channel 1 and channel 2 .

From the discussion so far, we note that the power allocation strategy between $\mathrm{CF}$ and $\mathrm{EF} / \mathrm{AF}$ are different. In $\mathrm{CF}$, the relay power and source power allocation are decoupled and we end up allocating more power to the better channel. On the other hand, in EF/AF, the source and relay power allocation affect and compensate each other to yield maximum rate.

So far, we have considered the cases where SR links are always better than SD links. Switching each received SNR of SR and SD for each channel, we have the same four cases as Table I except that SD links are now better than SR links.

Figure 8 shows the maximized achievable rates of three relay transmission schemes with optimum power allocation when SD links are better than SD links. Although the rates obtained are very close, we can note that $\mathrm{CF}$ is still the best scheme and AF performs better than EF.

Comparing (8) and (16), we observe that if SD received SNR is larger than SR received SNR, the instantaneous achievable rate of EF is smaller than AF. On the other hand, if $\mathrm{SR}$ received $\mathrm{SNR}$ is larger than $\mathrm{SD}$ received $\mathrm{SNR}$, the 


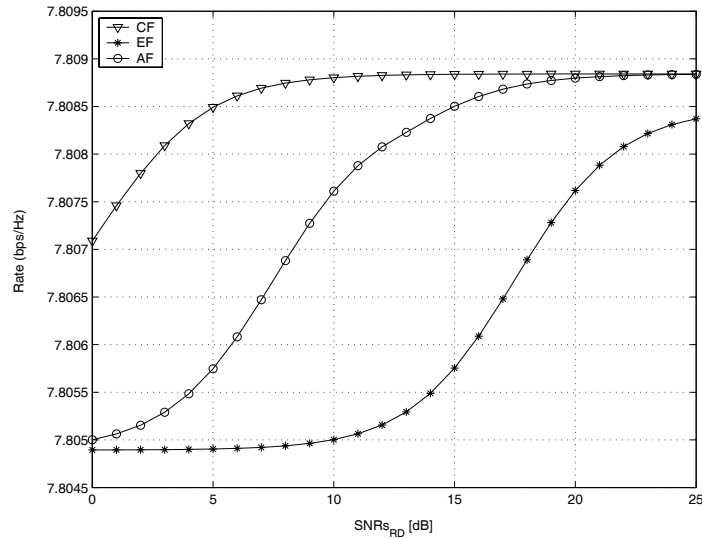

Fig. 8. Comparison of maximum achievable rates

instantaneous achievable rate of EF becomes the same as that of AF. The numerical results match with the observation. However, we must also note the existence of a recent result, [18], where the EF scheme based on unconstrained minimum mean square error (MMSE) estimate is shown to outperform the AF scheme based on linear estimate in memoryless relay networks.

\section{CONClusion}

In this paper, we have investigated the optimum source and relay power allocation problem for amplify-and-forward (AF), estimate-and-forward (EF), and compress-and-forward (CF) schemes for multi-band relay channels where the source node communicates to the destination with the help of a relay node via multiple orthogonal channels. For each scheme, we have proposed an iterative power allocation algorithm based on alternating minimization to maximize the achievable rate. For numerical results, we have considered different cases depending on channel conditions of the source to the relay (SR) and source to the destination (SD). It is shown that CF is always better than EF and AF. When SR links are better than SD links, EF and AF perform equally well. When SR links are worse than SD links, AF performs better than EF. We have observed that $\mathrm{CF}$ and EF/AF may yield different power allocation strategies. Proposed algorithms result in the optimum resource allocation, and thus can provide insight in choosing the best relay forwarding scheme under given channel conditions.

\section{REFERENCES}

[1] E. C. van der Meulen. Three terminal communications channels. Advanced Applied Probability, 3(5):120 - 154, September 1971.

[2] T. M. Cover and A. A. El Gamal. Capacity theorems for the relay channel. IEEE Transactions on Information Theory, 25(5):572 - 584, September 1979.

[3] A. Sendonaris, E. Erkip, and B. Aazhang. User cooperation diversity part I: System description; part II: Implementation, aspects and performance analysis. IEEE Transactions on Communications, 51(11):1927 - 1948, November 2003.

[4] A. Høst-Madsen and J. Zhang. Capacity bounds and power allocation in wireless relay channel. IEEE Transactions on Information Theory, 51(6):2020 - 2040, June 2005.
[5] M. Gastpar and M. Vetterli. On the capacity of large gaussian relay networks. IEEE Transactions on Information Theory, 51(3):765 - 779, March 2005.

[6] J. N. Laneman, D. N. C. Tse, and G. W. Wornell. Cooperative diversity in wireless networks: Efficient protocols and outage behavior. IEEE Transactions on Information Theory, 50(12):3062 - 3080, December 2004.

[7] Yindi Jing and Babak Hassibi. Cooperative diversity in wireless relay networks with multiple-antenna nodes. In IEEE International Symposium on Information Theory, ISIT 05', pages 815 - 819, September 2005.

[8] M. A. Khojastepour, A. Sabharwal, and B. Aazhang. On the capacity of cheap relay networks,. In Conference on Information Sciences and Systems, CISS' 03, March 2003.

[9] R. U. Nabar, H. Bölcskei, and F. W. Kneubühler. Fading relay channels: performance limits and space-time signal design. IEEE Journal on Selected Areas in Communications, 22(6):1099 - 1109, August 2004.

[10] J. N. Laneman, D. N. C. Tse, and G. W. Wornell. Distributed space-timecoded protocols for exploiting cooperative diversity in wireless networks. IEEE Transactions on Information Theory, 49(10):2415 - 2425, October 2003.

[11] N. Ahmed, M. A. Khojastepour, and B. Aazhang. Outage minimization and optimal power control for the fading relay channel,. In IEEE Information Theory Workshop, ITW' 04, pages 458 - 462, October 2004.

[12] Y. Liang and V. V. Veeravalli. Gaussian orthogonal relay channel: optimal resource allocation and capacity. IEEE Transactions on Information Theory, 2005, 51(9):3284 - 3289, September 2005.

[13] A. A. El Gamal, M. Mohseni, and S. Zahedi. On reliable communication over Additive White Gaussian Noise relay channels. Submitted to IEEE Transactions on Information Theory, September 2004.

[14] A. A. El Gamal and S. Zahedi. Capacity of a class of relay channels with orthogonal components. IEEE Transactions on Information Theory, 51(5):1815 - 1817, May 2005.

[15] K. Lee and A. Yener. On resource allocation for multi-band relay channel. In Conference on Information Sciences and Systems, CISS' 05, March 2005.

[16] I. Maric and R. D. Yates. Forwarding strategies for Gaussian parallelrelay networks. In Conference on Information Sciences and Systems, CISS’ 04, pages 591 - 596, March 2004.

[17] S. Serbetli and A. Yener. Power allocation and hybrid relaying strategies for f/tdma ad hoc networks. In International Conference on Wireless Networks, Communications, and Mobile Computing, WirelessCom' 05, June 2005.

[18] K. S. Gomadam and S. A. Jafar. On the capacity of memoryless relay networks. http://arxiv.org/list/cs.IT/0510.

[19] J. Luo, R. S. Blum, L. J. Cimini, L. J. Greenstein, and A. M. Haimovich. Decode-and-forward cooperative diversity with power allocation in wireless networks. In IEEE Global Telecommunications Conference, GLOBECOM’ 05, pages 3048 - 3052, 28 Nov.-2 Dec 2005.

[20] M. Chen, S. Serbetli, and A. Yener. Distributed power allocation for parallel relay networks. In IEEE Global Telecommunications Conference, GLOBECOM' 05, pages 1177 - 1181, 28 Nov.-2 Dec 2005.

[21] M. A. Khojastepour, A. Sabharwal, and B. Aazhang. Improved achievable rates for user cooperation and relay channels. In IEEE International Symposium on Information Theory, ISIT 04', page 4, July 2004.

[22] A. D. Wyner and J. Ziv. The rate-distortion function for source coding with side information at the decoder. IEEE Transactions on Information Theory, 22(1): 1 - 10, January 1976.

[23] G. Kramer, M. Gastpar, and P. Gupta. Cooperative strategies and capacity theorems for relay networks. IEEE Transactions on Information Theory, 51(9):3037 - 3063, September 2005.

[24] A. Vardy. Codes, Curves, and Signals: Common Threads in Communications, pages 173 - 191. Kluwer Academic Publishers, 1998. 